OPEN ACCESS

Edited by:

Lei Ye,

National Heart Centre Singapore,

Singapore

Reviewed by:

Jianhua Ma

Nanjing Medical University, China

Yuli Huang,

Southern Medical University, China

*Correspondence:

Weimin Cai

weimincai@fudan.edu.cn

Guang Ning

gning@sibs.ac.cn

YuXu

jane.yuxu@gmail.com

${ }^{\dagger}$ These authors have contributed equally to this work

Specialty section:

This article was submitted to

Clinical Diabetes,

a section of the journal

Frontiers in Endocrinology

Received: 26 October 2021

Accepted: 28 December 2021

Published: 26 January 2022

Citation:

Wang F, Zheng R, Li L, Xu M, Lu J,

Zhao Z, Li M, Wang T, Wang S,

Bi Y, Xu Y, Ning G and Cai W (2022) Novel Subgroups and Chronic Complications of Diabetes in MiddleAged and Elderly Chinese: A

Prospective Cohort Study.

Front. Endocrinol. 12:802114. doi: 10.3389/fendo.2021.802114

\section{Novel Subgroups and Chronic Complications of Diabetes in Middle-Aged and Elderly Chinese: A Prospective Cohort Study}

\author{
Fei Wang ${ }^{1,2,3 \dagger}$, Ruizhi Zheng ${ }^{2,3 \dagger}$, Ling $\mathrm{Li}^{2,3,4 \dagger}$, Min Xu ${ }^{2,3}$, Jieli Lu ${ }^{2,3}$, Zhiyun Zhao ${ }^{2,3}$, \\ Mian $L i^{2,3}$, Tiange Wang ${ }^{2,3}$, Shuangyuan Wang ${ }^{2,3}$, Yufang $B i^{2,3}, Y u X u^{2,3 *}$, \\ Guang Ning ${ }^{1,2,3 *}$ and Weimin Cai ${ }^{1 *}$ \\ ${ }^{1}$ Department of Clinical Pharmacy and Pharmaceutical Management, School of Pharmacy, Fudan University, \\ Shanghai, China, ${ }^{2}$ Department of Endocrine and Metabolic Diseases, Shanghai Institute of Endocrine and Metabolic \\ Diseases, Ruijin Hospital, Shanghai Jiao Tong University School of Medicine, Shanghai, China, ${ }^{3}$ Shanghai National Clinical \\ Research Center for Metabolic Diseases, Key Laboratory for Endocrine and Metabolic Diseases of the National Health \\ Commission of the PR China, Shanghai Key Laboratory for Endocrine Tumor, State Key Laboratory of Medical Genomics, \\ Ruijin Hospital, Shanghai Jiao Tong University School of Medicine, Shanghai, China, ${ }^{4}$ Department of Pharmacy, Ruijin \\ Hospital, Shanghai Jiao Tong University School of Medicine, Shanghai, China
}

Background: Diabetes mellitus, especially type 2 diabetes mellitus (T2DM), is regarded as highly heterogeneous. Novel diabetes phenotypes by cluster analysis have been proposed in Europeans but may show different cluster features in Asians. The applicability of cluster analysis in middle-aged and elderly Chinese community T2DM patients needs further investigation.

Methods: Participants were recruited from Jiading community in Shanghai, China. We adopted k-means cluster analysis in 1130 patients (aged $\geq 40$ years) with newlydiagnosed T2DM at baseline. Cluster analysis was performed based on seven variables, including fasting plasma glucose, 2 hours postprandial blood glucose, age at diagnosis, body mass index, hemoglobin A1c, homoeostatic model assessment estimates of $\beta$-cell function and insulin resistance. All subjects were re-examined at 4.4 years later. Metabolic associated fatty liver disease was diagnosed using B-ultrasound, hepatic fibrosis by non-invasive scores, renal and cardiovascular status by subclinical biomarkers. Multivariable logistic regression models were used to compare the risks of complications between clusters.

Results: Patients were classified into 4 clusters. 381 (33.7\%), 456 (40.4\%), 87 (7.7\%), and 206 (18.2\%) patients were separately assigned to mild age-related diabetes (MARD), mild obesity-related diabetes (MOD), severe insulin-deficient and insulin-resistant diabetes (SIDRD), or severe obesity-related and insulin-resistant diabetes (SOIRD), respectively. Participants in MARD, SOIRD, and SIDRD clusters were associated with significantly increased risks of different complications. SOIRD and SIDRD showed novel features in Chinese T2DM patients that were different from those in Europeans.

Conclusions: The refined diabetes phenotypic approach was applicable to Chinese middle-aged and elderly T2DM patients. Patients in different clusters presented 
significantly different characteristics, progression of metabolic features, and risks of diabetic complications.

Keywords: type 2 diabetes, cluster analysis, diabetic complications, metabolic associated fatty liver disease, subclinical atherosclerosis

\section{INTRODUCTION}

The number of adults with diabetes has progressively increased from 108 million in 1980 to 536.6 million in 2021 worldwide (1, 2). With a dramatic change in lifestyle in recent 30 years, China becomes the country with the largest number of patients affected by diabetes. A national survey in 2015-2017 showed that the prevalence of diabetes among Chinese people aged $\geq 18$ years was $12.8 \%$, but the rate of diabetes control was low (3). Poor control of blood glucose is associated with higher risk of incident diabetic complications, leading to a huge burden on the patients.

Nowadays, the most widely used diagnosis of diabetes is based on fasting and post-load glucose as well as hemoglobin A1c (HbAlc) levels defined by the American Diabetes Association (ADA) criteria (4). However, such criteria could not provide precision treatment recommendations for diabetes, which has heterogeneous physiopathology $(4,5)$. Recently, a novel approach has been proposed to classify diabetes into 5 subgroups with different characteristics (6): two mild subgroups with good metabolic control and few diabetesrelated complications, and three severe subgroups with poor glucose control and increased risks of clinical outcomes. Replications of novel diabetes subgroups have been observed in cohorts from north Europe, USA, and Asia (7-9). The Chinese population is more likely to have a fragile $\beta$-cell function and more susceptible to the effects of obesity on metabolic factors (10, 11). Chinese patients with type 2 diabetes mellitus (T2DM) present with different characteristics from those in other populations (12). Additionally, $2 \mathrm{~h}$ post-load plasma glucose (2hPG) is an important predictive factor of clinical outcomes in Chinese adults independent of fasting plasma glucose (FPG) and HbAlc (13), but 2hPG was not considered in any cluster analysis of diabetes in aforementioned studies $(6,14,15)$.

Furthermore, most reports were cross-sectional $(7,14)$ and few studies have examined such classification method among Chinese T2DM patients. In the current study, we aimed to examine the novel subgroups of diabetes in a cohort of middle-aged and elderly Chinese using variables reported in previous studies plus FPG and 2hPG at baseline (6), to evaluate the changes of specific metabolic markers during follow-up, and to assess the risks of developing diabetes-related complications among different clusters.

\section{METHODS}

\section{Study Design and Participants}

In the present study, participants with newly-diagnosed T2DM were included from a prospective cohort study of 10,375 adults aged $\geq 40$ years in Jiading District, the suburb of Shanghai,
China. The details of the study design have been reported previously $(16,17)$. Briefly, the baseline examination was conducted between March and August in 2010. Participants were interviewed face to face by well-trained staff with a comprehensive evaluation including a standard questionnaire, anthropometric measurements, blood and urine sampling, and biochemical determination according to a standard protocol. During August 2014 and May 2015, participants were invited for a follow-up examination to reassess their health conditions.

The study protocol was approved by the Institutional Review Board of Ruijin Hospital, Shanghai Jiaotong University School of Medicine. Informed consent was provided by each participant in advance.

\section{Data Collection}

A standard questionnaire including demographic characteristics, history of chronic diseases, medications, and lifestyle factors was administrated by a face-to-face interview. Current smoking and drinking were defined as smoking cigarettes or consuming alcohol regularly during past 6 months. Physical activity was inquired and evaluated using metabolic equivalent hours per week (MET-h/wk) by the short form of the International Physical Activity Questionnaire (IPAQ) (18). Being physically active was defined as at least $7.5 \mathrm{MET}-\mathrm{h} / \mathrm{wk}$ (19). Anthropometric measurements, including body height, weight, and waist and hip circumferences were measured according to a standard protocol. Body mass index (BMI) was calculated as body weight divided by height squared $\left(\mathrm{kg} / \mathrm{m}^{2}\right)$. Blood pressure (BP) was measured three times after at least 5-minute sitting rest using a calibrated automatic electronic device (OMRON Model HEM-752), with alcohol, tea, coffee, and exercise being strictly avoided 30 minutes before measurement. The average of 3 measurements was used for analysis. Blood samples were collected in early morning with an overnight fast for at least 10 hours. All participants without a diabetes history underwent a standard 75-g oral glucose tolerance test (OGTT), and blood samples were collected at $0 \mathrm{~h}$ and $2 \mathrm{~h}$. Plasma glucose was measured using the glucose oxidase method on an autoanalyzer (Modular P800; Roche, Basel, Switzerland). HbA1c was determined by high-performance liquid chromatography using the VARIANT II Hemoglobin Testing System (Bio-Rad Laboratories). Biochemical parameters including total cholesterol (TC), triglycerides (TG), low-density lipoprotein cholesterol (LDL-c), high-density lipoprotein cholesterol (HDL-c), alanine aminotransferase (ALT), aspartate aminotransferase (AST), $\gamma$-glutamyl transferase (GGT), apolipoprotein B (ApoB), apolipoprotein A1 (ApoA1) and albumin were measured with auto analyzers (Modular Analytics P800 and Modular E170; Roche, Basel, Switzerland). Serum creatinine (SCr) was measured using the picric acid 
method (clinical chemistry diagnostic system C16000, Abbott Laboratories, Otawarashi, Japan). A first void spot urine sample was collected in early morning to measure urinary albumin using immunoturbidimetric method (Beijing Atom High-Tech, Beijing, China) and urinary creatinine using Jaffe's kinetic method (Hitachi 7600-020, Tokyo, Japan).

Ankle-brachial index (ABI), brachial to ankle pulse wave velocity (ba-PWV), and carotid intima-media thickness (CIMT) were measured to evaluate subclinical atherosclerosis. The values of ABI and ba-PWV were obtained by Colin VP-1000 (Model BP203RPE II, form ABI/PWV). The ABI was calculated by the ratio of the dorsal foot or posterior tibial artery systolic BP to the brachial artery systolic BP. The ba-PWV was measured as pulse waves distances (obtained from the brachial and tibial arteries) divided by the transmission time. The CIMT was measured by high-resolution B-mode tomographic ultrasound system (Esaote Biomedica SpA, Italy) with a linear $7.5-\mathrm{MHz}$ transducer. The operator measured CIMT on the far wall of the common carotid arteries at $1.5 \mathrm{~cm}$ proximal to the bifurcation. The distance from the leading edge of the first echogenic line to the second at the end of diastole was taken for CIMT. The higher bilateral ba-PWV and CIMT value was used for analysis.

\section{Definitions of Diabetes and Other Complications}

Newly-diagnosed diabetes was defined by FPG $\geq 7.0 \mathrm{mmol} / \mathrm{L}$ $(126 \mathrm{mg} / \mathrm{dL})$ and/or $2 \mathrm{hPG} \geq 11.1 \mathrm{mmol} / \mathrm{L}$ (200 mg/dL) during OGTT and/or HbA1c $\geq 6.5 \%$ excluding self-reported diabetes or use of diabetes medications (4). Hypertension was defined by BP $\geq 140 / 90 \mathrm{mmHg}$ (20) and/or using antihypertensive medications within 2 weeks. Dyslipidemia was defined by TC $\geq 6.2 \mathrm{mmol} / \mathrm{L}$ $(240 \mathrm{mg} / \mathrm{dL})$ and/or LDL cholesterol $\geq 4.1 \mathrm{mmol} / \mathrm{L}(160 \mathrm{mg} / \mathrm{dL})$ and/or TG $\geq 2.3 \mathrm{mmol} / \mathrm{L}(200 \mathrm{mg} / \mathrm{dL})$ and/or HDL cholesterol $\leq$ $1.0 \mathrm{mmol} / \mathrm{L}$ (40 mg/dL) and/or taking lipid-lowering drugs (21). Obesity was defined by $\mathrm{BMI} \geq 25 \mathrm{~kg} / \mathrm{m}^{2}$. We estimated $\beta$-cell function by the homoeostatic model assessment estimates of $\beta$ cell function (HOMA- $\beta$ ) and insulin resistance by the homoeostatic model assessment estimates of insulin resistance (HOMA-IR) (22). Estimated glomerular filtration rate (eGFR) was calculated with serum creatinine levels using the Chronic Kidney Disease Epidemiology Collaboration (CKD-EPI) equation (23). Urinary albumin-creatinine ratio (ACR) was calculated by dividing urinary albumin concentrations $(\mathrm{mg} / \mathrm{L})$ by urinary creatinine concentrations $(\mathrm{g} / \mathrm{L})$. Chronic kidney disease $(\mathrm{CKD})$ was defined by eGFR $<60 \mathrm{ml} / \mathrm{min}$ per $1.73 \mathrm{~m}^{2}$ or ACR $\geq 30 \mathrm{mg} / \mathrm{g}$. Subclinical peripheral artery disease was defined as an ABI $\leq 1.0$ or $>1.3(24,25)$. Elevated CIMT $(\geq 0.7$ $\mathrm{mm})$ or ba-PWV ( $\geq 1991 \mathrm{~cm} / \mathrm{s})$ was defined as levels within the upper quartile of the study population at baseline.

Metabolic associated fatty liver disease (MAFLD) was defined by diabetes complicated with hepatic steatosis (26). Abdominal ultrasonography was performed to identify fatty liver in all the participants as recommended ${ }^{26}$ Two trained sonographers who were blinded to both clinical and laboratory data, operated the high-resolution B-mode tomographic ultrasound system (Esaote Biomedica $\mathrm{SpA}$ ) with a $3.5-\mathrm{MHz}$ probe. According to the international expert consensus statement in 2020 and Chinese Association for the Study of Liver Disease, fatty liver was diagnosed by the presence of at least two of the following findings: (1) diffusely increased echogenicity of the liver relative to the kidney, (2) ultrasound beam attenuation, or (3) poor visualization of intrahepatic structures (27). A third trained sonographer who was also blinded to this study would be asked if the diagnosis of fatty liver was contradicted between the previous two sonographers.

Noninvasive liver fibrosis scores including nonalcoholic fatty liver disease fibrosis score (NFS), Aspartate aminotransferase/ platelet ratio index (APRI) and Fibrosis-4 Index (FIB-4) were calculated with routine laboratory variables according to the equations listed below (28-30). The higher probability of fibrosis was defined as NFS $\geq-1.455$, FIB- $4 \geq 1.3$, or APRI $\geq 0.5$.

\section{Equations}

HOMA $-\beta=20 \times$ fasting insulin $(\mu \mathrm{lU} / \mathrm{mL}) /[$ fasting glucose $(\mathrm{mmol} / \mathrm{L})-3.5]$

HOMA - IR = fasting insulin $(\mu \mathrm{IU} / \mathrm{mL}) \times$ fasting glucose $(\mathrm{mmol} / \mathrm{L}) / 22.5$

NFS $=-1.675+0.037 \times$ age (years) $+0.094 \times$ BMI $\left(\mathrm{kg} / \mathrm{m}^{2}\right)+1.13$

$\times$ impaired fasting glucose or diabetes (yes $=1, \mathrm{no}=0$ ) +0.99

$\times$ AST $(\mathbf{U} / \mathbf{L}) /$ ALT $(\mathbf{U} / \mathbf{L})-0.013 \times$ platelet count $\left(\times 10^{9} / \mathbf{L}\right)-0.66 \times$ albumin $(\mathrm{g} / \mathrm{dL})$

APRI $=\left[(\right.$ AST $/$ normal upper limit AST $) /$ platelet count $\left(\times 10^{9} /\right.$ L $\left.)\right] \times 100$

FIB $-4=$ age $($ years $) \times$ AST $(U / L) /\left[\right.$ platelet count $\left.\left(\times 10^{9} / \mathbf{L}\right) \times \operatorname{ALT}(\mathbf{U} / \mathbf{L})^{1 / 2}\right]$

\section{Statistical Analysis}

In our study, K-means cluster analysis was performed with 7 variables including BMI, age at diagnosis of diabetes, FPG, 2hPG, HbA1c, HOMA- $\beta$, and HOMA-IR at baseline. The clustered variables are centered to means of 0 and standard deviations (SDs) of 1 . We replicated the clustering approach used by Ahlqvist et al. (6). The K-means cluster analysis was processed with a $\mathrm{k}$ value of 4 using the kmeansruns function (runs $=150$ ) in the fpc package in $\mathrm{R}$ version 4.0.3. We named the clusters based on the distinct cluster characteristics.

The continuous variables were presented in mean (standard deviation) or median ( $25 \%$ quartile, $75 \%$ quartile), and categorical variables were in numbers (\%). Comparisons of means and percentages were performed by the ANOVA and Chi-square tests between clusters, respectively. Bonferroni correction was applied to account for multiple comparisons. Skewed variables were log-transformed before analysis. Logistic regression analyses were done to compare the risks of incident diabetes-related complications between clusters, and the cluster with the lowest incidence of complications was used as the reference. The multivariable model was adjusted for sex, lifestyle (smoking status, drinking status, physical activity), family history of diabetes, education status, hypertension and dyslipidemia. Statistical analysis was performed with $\mathrm{R}$ version 4.0.3. We judged $P$ values less than 0.05 as statistical significance (2-sided). 


\section{RESULTS}

After excluding missing data on cluster variables $(\mathrm{n}=6)$, a total of 1130 newly-diagnosed T2DM participants were included for baseline cluster analysis. 688 in 1130 participants with complete cluster variable data underwent the follow-up interview. Participants with baseline self-reported disease history, missing data of biochemical or atherosclerotic cardiovascular measurements, or diabetes complications were further excluded for the association analysis (Supplementary Figure 1).

\section{Cluster Distribution and Characteristics at Baseline}

Participants were classified into 4 diabetes subgroups based on 7 variables measured at baseline (Figure 1 and Supplementary Material). They were categorized as mild age-related diabetes (MARD), mild obesity-related diabetes (MOD), severe insulindeficient and insulin-resistant diabetes (SIDRD) and severe obesity-related and insulin-resistant diabetes (SOIRD) respectively.

$381(33.7 \%)$ patients were assigned to the MARD cluster. Participants assigned to MARD had the oldest age at diagnosis (mean 68.8 years old), lowest BMI (mean $24.2 \mathrm{~kg} / \mathrm{m}^{2}$ ), and modest metabolic disturbances (mean HbAlc 6.2\%, median HOMA- $\beta$ 52.9, HOMA-IR 1.8). The MOD cluster makes up the largest proportion among diabetes patients $(n=456,40.4 \%)$. Individuals in the MOD cluster were characterized by younger age at diagnosis (mean 54.8 years old) and obesity (mean BMI $26.9 \mathrm{~kg} / \mathrm{m}^{2}$ ), but with moderate insulin release and insulin resistance status (median HOMA- $\beta$ 57.0, HOMA-IR 2.7). Patients in the SIDRD cluster $(\mathrm{n}=87,7.7 \%)$ manifested serious insulin deficiency and insulin resistance (median HOMA- $\beta$ 19.3, HOMA-IR 5.2), and the worst glucose control (mean HbAlc 10.6\%, FPG $13.0 \mathrm{mmol} / \mathrm{L}, 2 \mathrm{hPG} 25.4 \mathrm{mmol} / \mathrm{L}$ ). 206 (18.2\%) individuals were assigned to the SOIRD cluster who were characterized as having the highest BMI (mean $29.8 \mathrm{~kg} / \mathrm{m}^{2}$ ), the most serious insulin resistance, and the highest insulin release level (median HOMA- $\beta$ 148.9, HOMA-IR 5.6).

Anthropometric and clinical data of the participants at baseline are presented in Table $\mathbf{1}$ by clusters. Participants in the MARD cluster presented with the lowest proportion of family history of diabetes (8.7\%), lower level of LDL cholesterol (3.27 \pm $0.88 \mathrm{mmol} / \mathrm{L})$, TC $(5.51 \pm 1.01 \mathrm{mmol} / \mathrm{L})$ and TG (median 1.53 $\mathrm{mmol} / \mathrm{L})$, and the highest level of HDL cholesterol $(1.37 \pm 0.36$ $\mathrm{mmol} / \mathrm{L}$ ) than those in other clusters. Meanwhile, participants in the SIDRD cluster had higher proportion of smoking (41.3\%), drinking (35.6\%) than those of the other clusters and SIDRD was the only cluster with a larger proportion of male $(60.9 \%)$ than the other clusters.

\section{Risk of Developing Diabetes-Related Clinical/Subclinical Complications by Clusters}

Table 2 shows the incidences and risks of developing diabetesrelated complications at follow-up by clusters. During a median of 4.4 years, the risk of developing elevated ba-PWV was higher in cluster MARD (OR 2.60; 95\% CI 1.15-6.35) compared with
SOIRD. The risk of developing abnormal $\mathrm{ABI}$ was higher in cluster SOIRD (OR 3.46; 95\% CI 1.54-8.04) compared with MARD. Patients in MARD (OR 3.40; 95\% CI 1.64-7.41), SIDRD (OR 3.93; 95\% CI 1.40-10.58) and SOIRD (OR 2.70; 95\% CI 1.13-6.52) clusters all showed higher risks of CKD compared with MOD.

A total of $62(22.1 \%)$ patients developed MAFLD. The incidence of newly-diagnosed MAFLD at follow-up was $12.2 \%$, $30.6 \%, 15.0 \%$, and $43.8 \%$ in MARD, MOD, SIDRD, and SOIRD, respectively. Participants assigned to the MOD (OR 4.74; 95\% CI 2.19-10.86) and the SOIRD cluster (OR 5.06; 95\% CI 1.44-17.35) were significantly associated with higher risk of incident MAFLD compared with the MARD cluster. Compared with SIDRD, MARD was associated with an increased risk of liver fibrosis defined by FIB- $4 \geq 1.3$ (OR 4.10; 95\% CI 1.32-13.31).

\section{Changes in Clinical Metabolic Biomarker}

Figure 2 shows the changes of blood glucose, serum lipids, and blood pressure from baseline to follow-up by clusters. The levels of glycemic parameters (FPG, $2 \mathrm{hPG}$ and $\mathrm{HbAlc}$ ) were significantly decreased in SIDRD. The levels of FPG were significantly increased in the clusters of MARD, MOD and SOIRD, while $2 \mathrm{hPG}$ showed no significant change in these clusters after 4.4 years. TC and $\mathrm{ApoB} / \mathrm{ApoA} 1$ ratio were also decreased dramatically during the follow-up period (Supplementary Table 2). The SBP and DBP levels declined in all the clusters.

We then compared the changes of diabetic complicationsrelated metabolic biomarkers between clusters (Figure 3 and Supplementary Table 3). ACR and eGFR levels worsened in most of the clusters after 4.4 years. Patients assigned to SIDRD presented with the highest ACR level (median $11.0 \mathrm{mg} / \mathrm{g}$ at baseline and $14.9 \mathrm{mg} / \mathrm{g}$ at follow-up) while participants in MARD showed the lowest eGFR level both at baseline (80.1 \pm $\left.12.3 \mathrm{ml} / \mathrm{min} / 1.73 \mathrm{~m}^{2}\right)$ and follow-up $\left(75.8 \pm 13.2 \mathrm{ml} / \mathrm{min} / 1.73 \mathrm{~m}^{2}\right)$ compared with the other clusters (Figures 3A, B). Markers of liver fibrosis are shown in Figures 3C-E. The APRI, NFS, FIB4 scores mostly showed significantly increased trend in 4 clusters. After 4.4 years, the ba-PWV, ABI and CIMT increased significantly in SIDRD. The ba-PWV level also increased in MARD and MOD. The CIMT level increased in all the clusters (Figures 3F-H).

\section{DISCUSSION}

Using data from a prospective cohort study of Chinese community residents aged $\geq 40$ years, we were able to identify four subgroups of T2DM based on the 7 variables measured at baseline and used in the cluster analysis, including age at diagnosis, BMI, FPG, 2hPG, HbA1c, HOMA- $\beta$, and HOMAIR. Clusters were separated well in phenotypic characteristics and presented with different risks of complications. Our study had three key findings. Firstly, we found 2 clusters (SIDRD and SOIRD) which showed different features in Chinese T2DM from those in Ahlqvist-derived diabetes classifications in Caucasians $(6,9)$. 

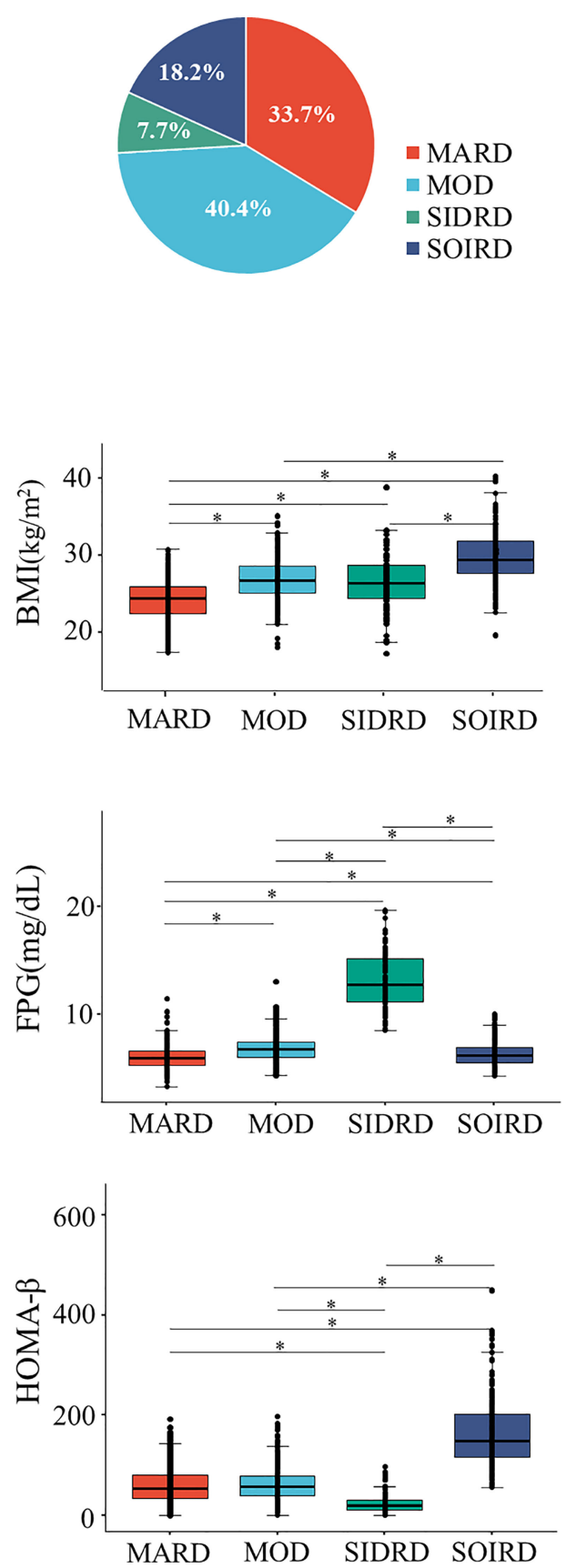
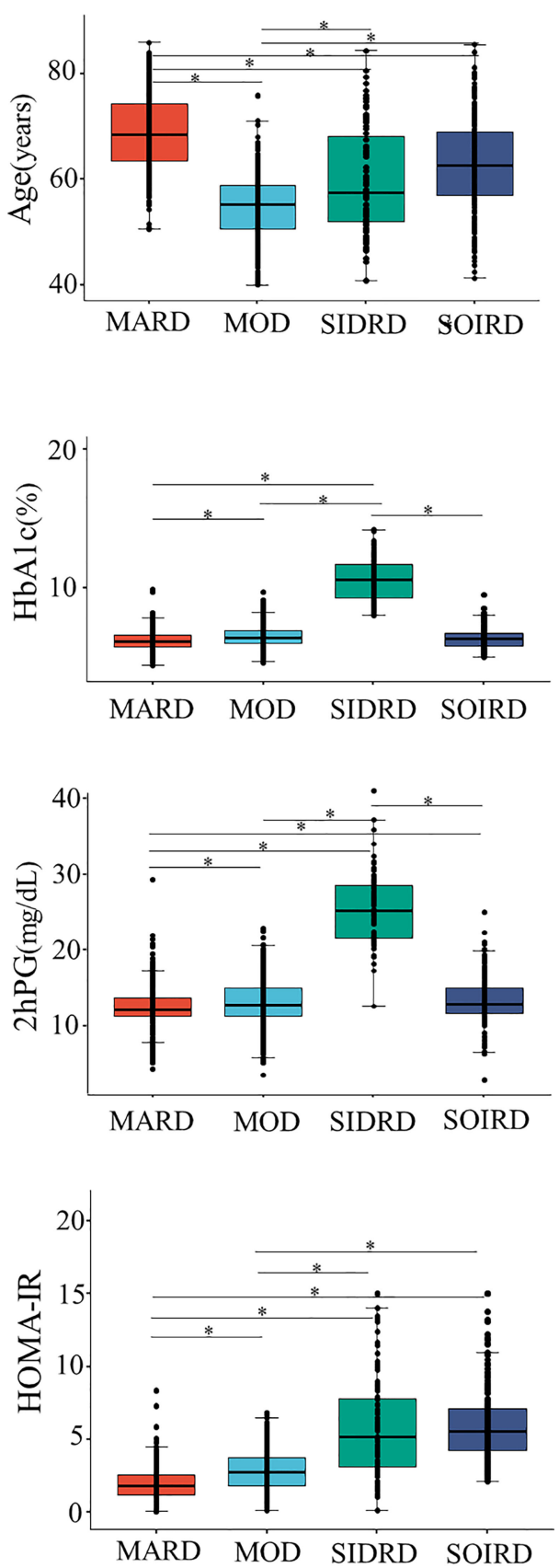

FIGURE 1 | Distributions and cluster characteristics at baseline. *The differences between clusters were compared by using t tests, and the Bonferroni correction was used to adjust the statistical significance level $(P=0.05 / 6)$. MARD, mild age-related diabetes; MOD, mild obesity-related diabetes; SIDRD, severe insulindeficient and insulin-resistant diabetes; SOIRD, severe obesity-related and insulin-resistant diabetes; BMI, body mass index; HbA1c, hemoglobin A1c; FPG, fasting plasma glucose; 2hPG, 2 hours postprandial blood glucose; HOMA- $\beta$, homoeostatic model assessment estimates of $\beta$-cell function; HOMA-IR, homoeostatic model assessment estimates of insulin resistance.

Secondly, after adjustment for multiple covariates, patients in different clusters presented significantly different risks of comprehensive diabetes-related complications. Thirdly, we observed different changes in subclinical metabolic biomarkers and outcome indexes among clusters as the disease progressed after a median of 4.4 years.
Compared with the current classification of diabetes, the novel classification proposed by Ahlqvist et al. in European populations had been verified in different populations and showed advantages in predicting diabetes progression and estimating risks of complications $(7-9,14,31)$. The $2 \mathrm{hPG}$ level is an important 
TABLE 1 | Patients' characteristics at baseline among the 4 clusters.

\begin{tabular}{|c|c|c|c|c|c|c|}
\hline Characteristics & Total & MARD & MOD & SIDRD & SOIRD & $P$ value \\
\hline No. of participants, n (\%) & $1130(100.0)$ & $381(33.7)$ & $456(40.4)$ & $87(7.7)$ & 206 (18.2) & $<0.001$ \\
\hline Age $(y r)$ & $61.4(9.6)$ & $68.8(7.2)$ & $54.8(6.1)$ & $59.9(10.2)$ & $62.9(9.0)$ & $<0.001$ \\
\hline Male, n (\%) & $466(41.2)$ & $158(41.5)$ & $196(43)$ & $53(60.9)$ & $59(28.6)$ & $<0.001$ \\
\hline $\mathrm{BMl}\left(\mathrm{kg} / \mathrm{m}^{2}\right)$ & $26.5(3.5)$ & $24.2(2.7)$ & $26.9(2.7)$ & $26.4(3.6)$ & $29.8(3.5)$ & $<0.001$ \\
\hline $\mathrm{FPG}(\mathrm{mmol} / \mathrm{L})$ & $7.0(2.2)$ & $6.1(1.0)$ & $6.9(1.2)$ & $13.0(2.7)$ & $6.4(1.1)$ & $<0.001$ \\
\hline $2 \mathrm{hPG}(\mathrm{mmol} / \mathrm{L})$ & $13.8(4.7)$ & $12.3(3.2)$ & $13.0(3.2)$ & $25.4(4.7)$ & $13.4(3.2)$ & $<0.001$ \\
\hline $\mathrm{HbA} 1 \mathrm{c}(\%)$ & $6.7(1.4)$ & $6.2(0.7)$ & $6.5(0.8)$ & $10.6(1.6)$ & $6.4(0.7)$ & $<0.001$ \\
\hline HOMA-IR & $2.8(1.7,4.2)$ & $1.8(1.2,2.6)$ & $2.7(1.8,3.8)$ & $5.2(3.1,7.8)$ & $5.6(4.2,7.1)$ & $<0.001$ \\
\hline HOMA- $\beta$ & $61.4(36.6,102.7)$ & $52.9(34.8,80.8)$ & $57.0(38.7,78.9)$ & $19.3(11.3,29.8)$ & $148.9(116.8,202.7)$ & $<0.001$ \\
\hline Smoking, n (\%) & $249(22.0)$ & $58(15.2)$ & $130(8.5)$ & $36(41.3)$ & $25(12.1)$ & $<0.001$ \\
\hline Drinking, $\mathrm{n}(\%)$ & $229(20.3)$ & $61(16.5)$ & $113(24.8)$ & $31(35.6)$ & $24(11.7)$ & $<0.001$ \\
\hline Physical activity (METs-h/wk) & $23.1(0.0,53.4)$ & $23.10(4.7,42.0)$ & $28.0(0.0,132.0)$ & $23.1(3.6,92.8)$ & $23.1(0.0,46.2)$ & $<0.001$ \\
\hline Family history of diabetes, $\mathrm{n}(\%)$ & $160(14.2)$ & $33(8.7)$ & $86(18.9)$ & $15(17.2)$ & $26(12.6)$ & 0.001 \\
\hline Systolic blood pressure (mmHg) & $149.0(19.4)$ & $149.3(18.1)$ & $146.8(19.7)$ & $149.3(19.1)$ & $151.5(20.9)$ & 0.027 \\
\hline Diastolic blood pressure $(\mathrm{mmHg})$ & $85.0(10.7)$ & $81.6(10.0)$ & $87.6(10.5)$ & $86.9(10.6)$ & $84.9(11.0)$ & $<0.001$ \\
\hline LDL cholesterol (mmol/L) & $3.33(0.91)$ & $3.27(0.88)$ & $3.33(0.90)$ & $3.39(1.10)$ & $3.41(0.90)$ & 0.345 \\
\hline HDL cholesterol (mmol/L) & $1.27(0.31)$ & $1.37(0.36)$ & $1.23(0.28)$ & $1.20(0.29)$ & $1.22(0.27)$ & $<0.001$ \\
\hline Total cholesterol (mmol/L) & $5.59(1.10)$ & $5.51(1.01)$ & $5.57(1.11)$ & $5.96(1.51)$ & $5.60(1.02)$ & 0.007 \\
\hline Triglycerides (mmol/L) & $1.75(1.26,2.27)$ & $1.53(1.11,2.07)$ & $1.79(1.24,2.46)$ & $2.23(1.48,3.37)$ & $2.00(1.54,2.58)$ & $<0.001$ \\
\hline
\end{tabular}

Data are expressed as mean (SD) for variables with normal distribution, median (IQR) for variables with skewed distribution, and $n$ (\%) for categorical variables.

BMl, body mass index; FPG, fasting plasma glucose; 2 PPG, 2 hours post-load plasma glucose; HbA1c, hemoglobin A1c; HOMA- $\beta$, homoeostatic model assessment estimates of $\beta$-cell function; HOMA-IR, homoeostatic model assessment estimates of insulin resistance; LDL, low-density lipoprotein; HDL, high-density lipoprotein; MARD, mild age-related diabetes; MOD, mild obesity-related diabetes; SIDRD, severe insulin-deficient and insulin-resistant diabetes; SOIRD, severe obesity-related and insulin-resistant diabetes.

TABLE 2 | Risks of diabetes-related complications in 4 clusters at follow-up.

\begin{tabular}{|c|c|c|c|c|}
\hline & \multirow[t]{2}{*}{$\mathbf{N}$} & \multirow[t]{2}{*}{ n (\%) } & \multicolumn{2}{|c|}{ OR $(95 \% \mathrm{Cl})$} \\
\hline & & & Model 1 & Model 2 \\
\hline \multicolumn{5}{|c|}{ ba-PWV $\geq 1991(\mathrm{~cm} / \mathrm{s})$} \\
\hline MARD & 126 & $28(22.2)$ & $2.26(1.01-5.44)$ & $2.60(1.15-6.35)^{\star}$ \\
\hline MOD & 241 & $28(11.6)$ & $1.10(0.50-2.65)$ & $1.21(0.54-2.93)$ \\
\hline SIDRD & 37 & $8(216)$ & $2.45(0.82-7.28)$ & $2.85(0.94-8.59)$ \\
\hline SOIRD & 84 & $9(10.7)$ & Reference & Reference \\
\hline \multicolumn{5}{|c|}{$A B \mid \leq 1.0$ or $>1.3$} \\
\hline MARD & 162 & $13(8.0)$ & Reference & Reference \\
\hline MOD & 229 & $19(8.3)$ & $0.98(0.44-2.25)$ & $1.00(0.45-2.30)$ \\
\hline SIDRD & 47 & $4(8.5)$ & $1.05(0.28-3.29)$ & $1.08(0.28-3.41)$ \\
\hline SOIRD & 91 & $19(20.9)$ & $3.41(1.55-7.79)^{\star \star}$ & $3.46(1.54-8.04)^{\star *}$ \\
\hline \multicolumn{5}{|c|}{$\mathrm{CIMT} \geq 0.7(\mathrm{~mm})$} \\
\hline MARD & 137 & $93(67.9)$ & $0.99(0.60-1.63)$ & $0.99(0.60-1.63)$ \\
\hline MOD & 239 & $160(66.9)$ & Reference & Reference \\
\hline SIDRD & 45 & $31(68.9)$ & $0.98(0.49-2.05)$ & $0.97(0.48-2.04)$ \\
\hline SOIRD & 84 & $61(72.6)$ & $1.24(0.70-2.25)$ & $1.25(0.70-2.28)$ \\
\hline \multicolumn{5}{|c|}{$\mathrm{ACR} \geq 30(\mathrm{mg} / \mathrm{g})$ or eGFR $<60\left(\mathrm{ml} / \mathrm{min} / 1.73 \mathrm{~m}^{2}\right)$} \\
\hline MARD & 160 & $31(19.4)$ & $3.37(1.63-7.33)^{\star \star}$ & $3.40(1.64-7.41)^{\star \star}$ \\
\hline MOD & 217 & $15(6.9)$ & Reference & Reference \\
\hline SIDRD & 44 & $10(22.7)$ & $4.00(1.44-10.72)^{\star \star}$ & $3.93(1.40-10.58)^{\star \star}$ \\
\hline SOIRD & 86 & $14(16.3)$ & $2.85(1.19-6.86)^{\star}$ & $2.70(1.13-6.52)^{\star}$ \\
\hline \multicolumn{5}{|l|}{ MAFLD } \\
\hline MARD & 123 & $15(12.2)$ & Reference & Reference \\
\hline MOD & 121 & $37(30.6)$ & $4.45(2.07-10.10)^{\star \star \star}$ & $4.74(2.19-10.86)^{\star \star \star \star}$ \\
\hline SIDRD & 20 & $3(15.0)$ & $1.09(0.16-4.63)$ & $1.10(0.16-4.75)$ \\
\hline SOIRD & 16 & $7(43.8)$ & $5.20(1.48-17.82)^{\star *}$ & $5.06(1.44-17.35)^{\star \star}$ \\
\hline \multicolumn{5}{|l|}{$\mathrm{FIB}-4 \geq 1.3$} \\
\hline MARD & 60 & $48(80.0)$ & $4.11(1.36-12.95)^{\star}$ & $4.10(1.32-13.31)^{*}$ \\
\hline MOD & 156 & $87(55.8)$ & $1.26(0.51-3.13)$ & $1.23(0.48-3.12)$ \\
\hline SIDRD & 25 & $13(52.0)$ & Reference & Reference \\
\hline SOIRD & 39 & $25(64.1)$ & $1.76(0.57-5.55)$ & $1.90(0.60-6.14)$ \\
\hline \multicolumn{5}{|l|}{ APRI $\geq 0.5$} \\
\hline MARD & 183 & $30(16.4)$ & $1.14(0.63-2.04)$ & $1.11(0.61-1.99)$ \\
\hline$M O D$ & 253 & $38(15.0)$ & Reference & Reference \\
\hline SIDRD & 46 & $7(15.2)$ & $1.12(0.42-2.64)$ & $1.20(0.45-2.86)$ \\
\hline SOIRD & 79 & $16(20.3)$ & $1.43(0.67-2.92)$ & $1.50(0.70-3.09)$ \\
\hline \multicolumn{5}{|c|}{$N F S \geq-1.455$} \\
\hline MARD & 35 & $26(74.3)$ & $1.06(0.41-2.84)$ & $0.93(0.35-2.55)$ \\
\hline MOD & 91 & 60 (65.9) & Reference & Reference \\
\hline SIDRD & 18 & $14(77.8)$ & $1.66(0.51-6.47)$ & $2.11(0.63-8.56)$ \\
\hline SOIRD & 23 & $16(69.6)$ & $1.14(0.39-3.72)$ & $1.31(0.44-4.30)$ \\
\hline
\end{tabular}

${ }^{* * *} p<0.001,{ }^{* *} p<0.01,{ }^{*} p<0.05$.

$N$, the number of individuals in the cluster at follow-up; $n$, the number of individuals with clinical/subclinical outcomes.

Model 1 was adjusted for gender, lifestyle (including smoking status, drinking status, physical activity), family history of diabetes, and education status. Model 2 was further adjusted for hypertension and dyslipidaemia.

OR, odds ratio; CI, confidence interval; MAFLD, metabolic associated fatty liver disease; FIB-4, fibrosis 4 score; ACR, albumin-to-creatinine ratio; eGFR, estimated glomerular filtration rate; ba-PWV, brachial-ankle pulse wave conduction velocity; ABI, ankle brachial index; CIMT, carotid intima - media thickness; MARD, mild age-related diabetes; MOD, mild obesity-related diabetes; SIDRD, severe insulin-deficient and insulin-resistant diabetes; SOIRD, severe obesity and insulin-resistant diabetes. 
A

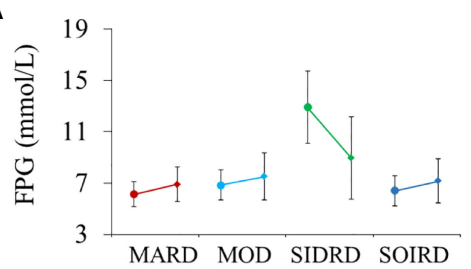

D

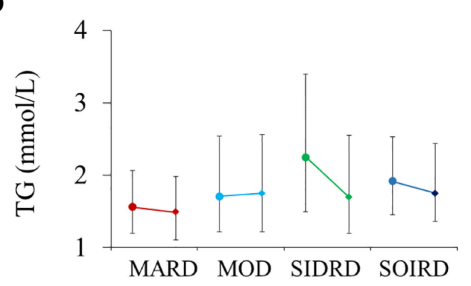

G

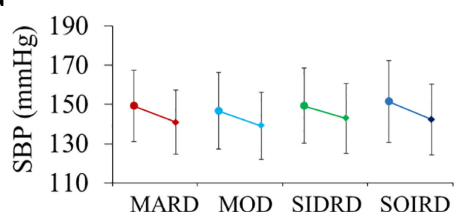

B

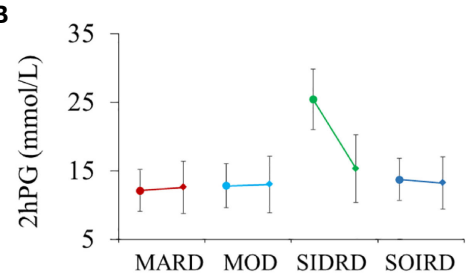

E

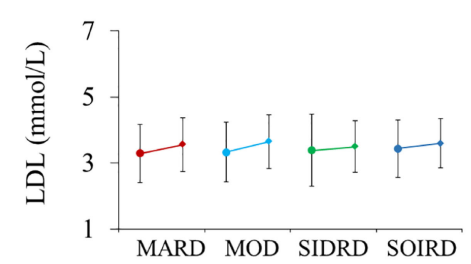

H

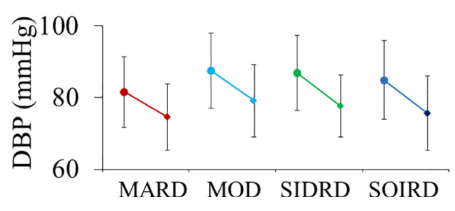

C

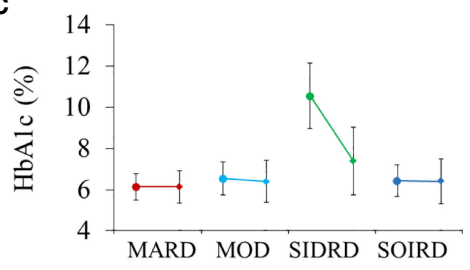

$\mathbf{F}$

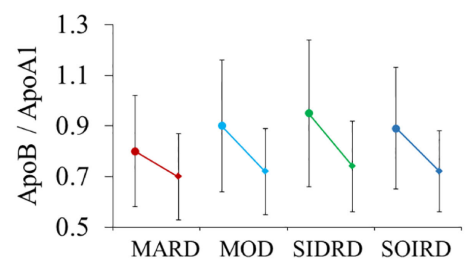

FIGURE 2 | Changes in the metabolic biomarkers during follow-up by clusters. Plots show levels of FPG (A), 2hPG (B), HbA1c (C), TG (D), LDL (E), ApoB/ApoA1 $\mathbf{( F )}$, SBP (G), and DBP (H). Levels are shown for patients with data available at both baseline (circles) and follow-up (diamond). Data are expressed as mean (SD) for variables with normal distribution or median (IQR) for variables with skewed distribution. $P$ values for paired t-test between baseline and follow-up by clusters are presented in Supplementary Table 2. MARD, mild age-related diabetes; MOD, mild obesity-related diabetes; SIDRD, severe insulin-deficient and insulin-resistant diabetes; SOIRD, severe obesity-related and insulin-resistant diabetes; FPG, fasting plasma glucose; 2hPG, 2 hours postprandial blood glucose; HbA1c, hemoglobin A1c; TG, triglyceride; LDL, low density lipoprotein; ApoB, apolipoprotein B; ApoA1, apolipoprotein A1; SBP, systolic blood pressure; DBP, diastolic blood pressure.

marker for complications in T2DM. Chinese diet is characterized by a higher consumption of carbohydrate-rich cereals, accounting for $60 \%$ of the glycemic load (32). Furthermore, our previous study found that the $2 \mathrm{hPG}$ level is predictive of diabetes and complications in Chinese adults, independent of FPG and HbAlc (13). However, 2hPG was not included in cluster analysis in previous studies $(6,7,14)$. When $2 \mathrm{hPG}$ was taken into account in the current study, the SIDRD cluster had a substantially higher 2hPG at diagnosis and at follow-up, and had higher risks of developing CKD than the other clusters. It would be helpful to target these individuals with intensified treatment to reduce $2 \mathrm{hPG}$ level and prevent diabetic complications.

Characteristics of the clusters identified in our study showed some differences from those in the Swedish cohort (6), especially the SIDRD and SOIRD clusters. The SIDRD cluster presented with combined insulin deficiency and insulin resistance. The SOIRD cluster was featured as insulin resistant and obese which was different from insulin-resistant SIRD cluster as reported by previous studies $(6,7,9)$. The presentation of both insulin-resistant and insulin-deficient features in SIDRD cluster in East Asians, including Chinese, may be due to the ethnic characteristics of Asians with a lower capacity of insulin secretion and a rapidly increased insulin resistance compared to western populations $(33,34)$.

CVD is the major cause of morbidity and mortality in T2DM, and even in prediabetes $(35,36)$. Pathophysiological changes involved in the development of CVD in T2DM patients included myocardial insulin resistance, mitophagy, oxidative stress, apoptosis, and inflammation (37-39). The associations between different diabetes clusters and risks of macrovascular and microvascular complications had been reported $(6,9)$. However, little is known about the relationship between novel diabetes subgroups and the progression of subclinical atherosclerosis. Ba-PWV is an indicator of arterial stiffness and is an independent predictor of CVD risks (40). In the present study, we found that the cluster of MARD was associated with a higher risk of developing abnormal ba-PWV. Moreover, the higher risk of abnormal ABI in the SOIRD cluster indicated that age and insulin resistance might play an important role in the development of CVD (41).

We observed that patients in the SIDRD cluster had the highest risk of developing $\mathrm{CKD}$, while in previous studies the highest risk was in the SIRD cluster accompanied with serious insulin resistance $(6,9)$. Serious insulin resistance and insulin deficiency in SIDRD contribute to the development of CKD. Insulin resistance was associated with diabetic kidney disease regardless of the HbAlc level (6), while the insulin deficiency speeds up the development of CKD.

Non-alcoholic fatty liver disease (NAFLD) is associated with an increased risk of diabetes, CKD, and cardiovascular disease (42). In 2019, a consensus of international experts recommended 


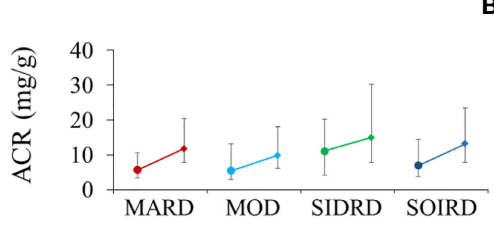

C

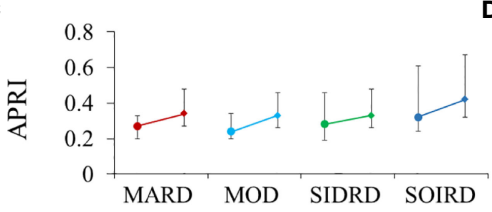

B

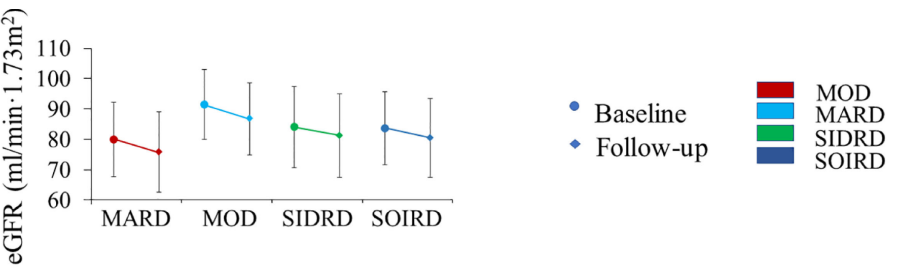

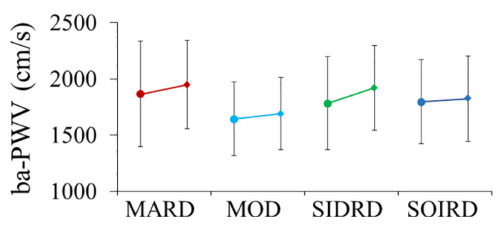
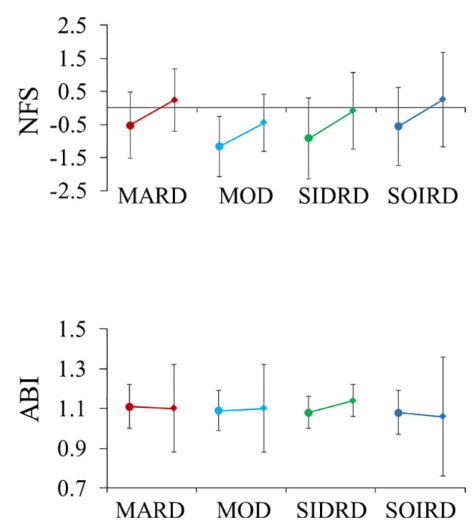

E

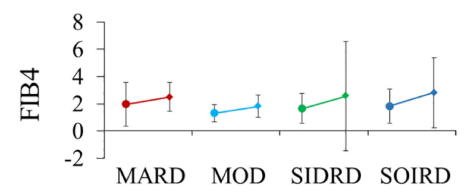

H

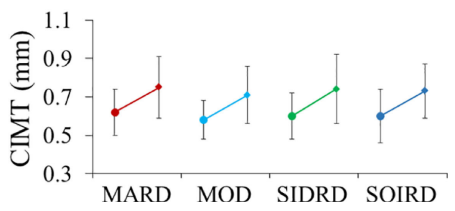

FIGURE 3 | The progression of chronic complications during follow-up by clusters. Plots show levels of ACR (A), eGFR (B), APRI (C), NFS (D), FIB4 (E), ba-PWV (F), ABI (G), and CIMT (H). Levels are shown for patients with data available at both baseline (circles) and follow-up (diamond). Data are expressed as mean (SD) for variables with normal distribution or median (IQR) for variables with skewed distribution. $P$ values for paired t-test between baseline and follow-up by clusters are presented in Supplementary Table 3. MARD, mild age-related diabetes; MOD, mild obesity-related diabetes; SIDRD, severe insulin-deficient and insulin-resistant diabetes; SOIRD, severe obesity-related and insulin-resistant diabetes. ACR, urinary albumin-creatinine ratio; eGFR, Estimated glomerular filtration rate; APRI, aspartate amino transferase/platelet ratio index; NFS, fatty liver disease fibrosis score; FIB4, fibrosis-4 index; ba-PWV, brachial to ankle pulse wave velocity; ABI, ankle-brachial index; CIMT, carotid intima-media thickness.

to use metabolic (dysfunction) associated MAFLD to raise the awareness of the fatty liver disease (26). Our study benefited from the use of B-ultrasound to diagnose MAFLD in diabetes patients. We found obesity-related clusters, including MOD and SOIRD, had higher risks of developing MAFLD compared with the other clusters. Whereas, in the study of Ahlqvist et al., the NAFLD was associated with SIRD but not with MOD (6). This might due to the cross-ethnic differences and that Chinese people generally had higher amounts of visceral fat than White people (43). Furthermore, non-invasive liver fibrosis indexes have been used to stratify the risk of liver-related morbidity and mortality in MAFLD patients with comparable performance to a liver biopsy. The MARD cluster had the highest risk of liver fibrosis observed by FIB-4 in our study, indicating that patients with MAFLD in the MARD cluster should pay more attention to monitoring liver fibrosis.

Our study has several limitations. Firstly, parameters such as the glutamic acid decarboxylase antibodies (GADA) were not measured or included in the cluster analysis in the current study. However, the prevalence of being GADA-positive could be less than $5.9 \%$ in population-based screening of adult-onset T2DM in China (7). Secondly, the follow-up duration was short and the limited numbers of incident macrovascular and microvascular diseases might not be able to provide sufficient statistical power to detect a true difference, therefore a further examination of these diabetic complications were not conducted. Thirdly, our study was conducted in community residents in Shanghai, China, which had limited extensibility to other populations.

In conclusion, the middle-aged and elderly Chinese adults with newly-diagnosed diabetes can be allocated to specific clusters. Two subgroups SIDRD and SOIRD in the current study showed higher risks of developing diabetes-related complications. In addition, obesity-related groups of MOD and SOIRD presented with a higher incidence of MAFLD. The more precise classifications of Chinese diabetes patients can aid in precision treatment of diabetes and prevention of complications.

\section{DATA AVAILABILITY STATEMENT}

The IRB has requested that currently, the dataset should be used by the research team members only. If the dataset has to be accessed to verify the results, the request can be directed to the corresponding author. Requests to access the datasets should be directed to Yu Xu, jane.yuxu@gmail.com.

\section{ETHICS STATEMENT}

The studies involving human participants were reviewed and approved by The Institutional Review Board of Ruijin Hospital, 
Shanghai Jiaotong University School of Medicine. The patients/ participants provided their written informed consent to participate in this study.

\section{AUTHOR CONTRIBUTIONS}

FW, RZ, LL, YX, GN, and WC conceived and designed the study. FW, RZ, and LL analyzed and interpreted the data. FW and RZ drafted the manuscript. YX, GN, and WC revised it. MX, JL, ZZ, ML, TW, SW, YB, and YX collected data. FW, RZ, and YX are the guarantors of this work and, as such, had full access to all the data in the study and take responsibility for the integrity of the data and the accuracy of the data analysis. All authors agreed to be accountable for all aspects of the work and approved the final version of the paper.

\section{FUNDING}

This work was supported by the grants from the National Natural Science Foundation of China (81870560, 81700764,

\section{REFERENCES}

1. NCD Risk Factor Collaboration. Worldwide Trends in Diabetes Since 1980: A Pooled Analysis of 751 Population-Based Studies With 4.4 Million Participants. Lancet (2016) 387(10027):1513-30. doi: 10.1016/S0140-6736 (16)00618-8

2. Sun H, Saeedi P, Karuranga S, Pinkepank M, Ogurtsova K, Duncan BB, et al. IDF Diabetes Atlas: Global, Regional and Country-Level Diabetes Prevalence Estimates for 2021 and Projections for 2045. Diabetes Res Clin Pract (2021) 24:109119. doi: 10.1016/j.diabres.2021.109119

3. Li Y, Teng D, Shi X, Qin G, Qin Y, Quan H, et al. Prevalence of Diabetes Recorded in Mainland China Using 2018 Diagnostic Criteria From the American Diabetes Association: National Cross Sectional Study. BMJ (2020) 369:m997. doi: 10.1136/bmj.m997

4. American Diabetes A. 2. Classification and Diagnosis of Diabetes: Standards of Medical Care in Diabetes-2021. Diabetes Care (2021) 44(Suppl 1):S15-33. doi: $10.2337 / \mathrm{dc} 21-\mathrm{S} 002$

5. Udler MS, Kim J, von Grotthuss M, Bonàs-Guarch S, Cole JB, Chiou J, et al. Type 2 Diabetes Genetic Loci Informed by Multi-Trait Associations Point to Disease Mechanisms and Subtypes: A Soft Clustering Analysis. PLoS Med (2018) 15(9):e1002654. doi: 10.1371/journal.pmed.1002654

6. Ahlqvist E, Storm P, Karajamaki A, Martinell M, Dorkhan M, Carlsson A, et al. Novel Subgroups of Adult-Onset Diabetes and Their Association With Outcomes: A Data-Driven Cluster Analysis of Six Variables. Lancet Diabetes Endocrinol (2018) 6(5):361-9. doi: 10.1016/S2213-8587(18)30051-2

7. Zou X, Zhou X, Zhu Z, Ji L. Novel Subgroups of Patients With Adult-Onset Diabetes in Chinese and US Populations. Lancet Diabetes Endocrinol (2019) 7 (1):9-11. doi: 10.1016/S2213-8587(18)30316-4

8. Xiong XF, Yang Y, Wei L, Xiao Y, Li L, Sun L. Identification of Two Novel Subgroups in Patients With Diabetes Mellitus and Their Association With Clinical Outcomes: A Two-Step Cluster Analysis. J Diabetes Investig (2021) 12 (8):1346-58. doi: 10.1111/jdi.13494

9. Zaharia OP, Strassburger K, Strom A, Bönhof GJ, Karusheva Y, Antoniou s, et al. Risk of Diabetes-Associated Diseases in Subgroups of Patients With Recent-Onset Diabetes: A 5-Year Follow-Up Study. Lancet Diabetes Endocrinol (2019) 7(9):684-94. doi: 10.1016/S2213-8587(19)30187-1

10. Wang T, Lu J, Shi L, Chen G, Xu M, Xu Y, et al. Association of Insulin Resistance and Beta-Cell Dysfunction With Incident Diabetes Among Adults in China: A Nationwide, Population-Based, Prospective Cohort Study. Lancet Diabetes Endocrinol (2020) 8(2):115-24. doi: 10.1016/ S2213-8587(19)30425-5
81941017, 81770842), the Shanghai Municipal Government (18411951800), Chinese Academy of Medical Sciences (2018PT32017, 2019PT330006), the Shanghai Shenkang Hospital Development Center (SHDC12019101, SHDC2020CR1001A, SHDC2020CR3069B), the Shanghai Jiaotong University School of Medicine (DLY201801), and the Ruijin Hospital (2018CR002).

\section{ACKNOWLEDGMENTS}

We acknowledge the dedication of all Jiading cohort study participants without whom this research would not be possible.

\section{SUPPLEMENTARY MATERIAL}

The Supplementary Material for this article can be found online at: https://www.frontiersin.org/articles/10.3389/fendo.2021.802114/ full\#supplementary-material

11. Zheng R, Li M, Xu M, Lu J, Wang T, Dai M, et al. Chinese Adults Are More Susceptible to Effects of Overall Obesity and Fat Distribution on Cardiometabolic Risk Factors. J Clin Endocrinol Metab (2021) 106(7): e2775-e88. doi: 10.1210/clinem/dgab049

12. Guideline for the Prevention and Treatment of Type 2 Diabetes Mellitus in China (2020 Edition) (in Chinese). Chin J Diabetes Mellitus (2021) 13(4):315409. doi: 10.19538/j.nk2021080106

13. Lu J, He J, Li M, Tang X, Hu R, Shi L, et al. Predictive Value of Fasting Glucose, Postload Glucose, and Hemoglobin A1c on Risk of Diabetes and Complications in Chinese Adults. Diabetes Care (2019) 42(8):1539-48. doi: $10.2337 / \mathrm{dc} 18-1390$

14. Wang W, Pei X, Zhang L, Chen Z, Lin D, Duan X, et al. Application of New International Classification of Adult-Onset Diabetes in Chinese Inpatients With Diabetes Mellitus. Diabetes Metab Res Rev (2020) 37(7):e3427. doi: 10.1002/dmrr.3427

15. Bello-Chavolla OY, Bahena-Lopez JP, Vargas-Vazquez A, Antonio-Villa NE, Márquez-Salinas A, Fermín-Martínez CA, et al. Clinical Characterization of Data-Driven Diabetes Subgroups in Mexicans Using a Reproducible Machine Learning Approach. BMJ Open Diabetes Res Care (2020) 8(1):e001550. doi: 10.1136/bmjdrc-2020-001550

16. Li M, Xu Y, Xu M, Ma L, Wang T, Liu Y, et al. Association Between Nonalcoholic Fatty Liver Disease (NAFLD) and Osteoporotic Fracture in Middle-Aged and Elderly Chinese. J Clin Endocrinol Metab (2012) 97 (6):2033-8. doi: 10.1210/jc.2011-3010

17. Wang L, Niu JY, Zhao ZY, Li M, Xu M, Lu JL, et al. Ideal Cardiovascular Health is Inversely Associated With Subclinical Atherosclerosis: A Prospective Analysis. BioMed Environ Sci (2019) 32(4):260-71. doi: 10.3967/bes2019.036

18. Craig CL, Marshall AL, Sjostrom M, Bauman AE, Booth ML, Ainsworth BE, et al. International Physical Activity Questionnaire: 12-Country Reliability and Validity. Med Sci sports Exercise (2003) 35(8):1381-95. doi: 10.1249/ 01.MSS.0000078924.61453.FB

19. U.S. Department of Health and Human Services. 2008 Physical Activity Guidelines for Americans. Washington, DC: US Department of Health and Human Services (2008). Available at: https://health.gov/paguidelines/pdf/paguide.pdf.

20. Whelton PK, Carey RM, Aronow WS, Casey DE Jr, Collins KJ, Dennison Himmelfarb C, et al. 2017 ACC/AHA/AAPA/ABC/ACPM/AGS/APhA/ASH/ ASPC/NMA/PCNA Guideline for the Prevention, Detection, Evaluation, and Management of High Blood Pressure in Adults: A Report of the American College of Cardiology/American Heart Association Task Force on Clinical Practice Guidelines. Hypertension (2018) 71(6):e13-e115. doi: 10.22141/23071257.7.1.2018.122220 
21. Joint Committee for Guideline R. 2016 Chinese Guidelines for the Management of Dyslipidemia in Adults. J Geriatr Cardiol (2018) 15(1):129. doi: 10.11909/j.issn.1671-5411.2018.01.011

22. Matthews DR, Hosker JP, Rudenski AS, Naylor BA, Treacher DF, Turner RC. Homeostasis Model Assessment: Insulin Resistance and Beta-Cell Function From Fasting Plasma Glucose and Insulin Concentrations in Man. Diabetologia (1985) 28(7):412-9. doi: 10.1007/BF00280883

23. Levey AS, Stevens LA, Schmid CH, Zhang YL, Castro AF 3rd, Feldman HI, et al. A New Equation to Estimate Glomerular Filtration Rate. Ann Internal Med (2009) 150(9):604-12. doi: 10.7326/0003-4819-150-9-200905050-00006

24. McDermott MM, Liu K, Criqui MH, Ruth K, Goff D, Saad MF, et al. AnkleBrachial Index and Subclinical Cardiac and Carotid Disease: The Multi-Ethnic Study of Atherosclerosis. Am J Epidemiol (2005) 162(1):33-41. doi: 10.1093/ aje/kwil67

25. Ohkuma T, Ninomiya T, Tomiyama H, Kario K, Hoshide S, Kita Y, et al. Ankle-Brachial Index Measured by Oscillometry is Predictive for Cardiovascular Disease and Premature Death in the Japanese Population: An Individual Participant Data Meta-Analysis. Atherosclerosis (2018) 275:141-8. doi: 10.1016/j.atherosclerosis.2018.05.048

26. Eslam M, Newsome PN, Sarin SK, Anstee QM, Targher G, Romero-Gomez M, et al. A New Definition for Metabolic Dysfunction-Associated Fatty Liver Disease: An International Expert Consensus Statement. J Hepatol (2020) 73 (1):202-9. doi: 10.1016/j.jhep.2020.03.039

27. Fan JG, Jia JD, Li YM, Wang BY, Lu LG, Shi JP, et al. Guidelines for the Diagnosis and Management of Nonalcoholic Fatty Liver Disease: Update 2010: (Published in Chinese on Chinese Journal of Hepatology 2010; 18:163166). J Dig Dis (2011) 12(1):38-44. doi: 10.1111/j.1751-2980.2010.00476.x

28. Huang X, Xu M, Chen Y, Peng K, Huang Y, Wang P, et al. Validation of the Fatty Liver Index for Nonalcoholic Fatty Liver Disease in Middle-Aged and Elderly Chinese. Med (Baltimore) (2015) 94(40):e1682. doi: 10.1097/ MD.0000000000001682

29. Angulo P, Hui JM, Marchesini G, Bugianesi E, George J, Farrell GC, et al. The NAFLD Fibrosis Score: A Noninvasive System That Identifies Liver Fibrosis in Patients With NAFLD. Hepatology (2007) 45(4):846-54. doi: 10.1002/ hep. 21496

30. Sterling RK, Lissen E, Clumeck N, Sola R, Correa MC, Montaner J, et al. Development of a Simple Noninvasive Index to Predict Significant Fibrosis in Patients With HIV/HCV Coinfection. Hepatology (2006) 43(6):1317-25. doi: 10.1002/hep. 21178

31. Anjana RM, Baskar V, Nair ATN, Jebarani S, Siddiqui MK, Pradeepa R, et al. Novel Subgroups of Type 2 Diabetes and Their Association With Microvascular Outcomes in an Asian Indian Population: A Data-Driven Cluster Analysis: The INSPIRED Study. BMJ Open Diabetes Res Care (2020) 8(1):e001506. doi: 10.1136/bmjdrc-2020-001506

32. Hu EA, Pan A, Malik V, Sun Q. White Rice Consumption and Risk of Type 2 Diabetes: Meta-Analysis and Systematic Review. BMJ (2012) 344:e1454. doi: 10.1136/bmj.e1454

33. Kodama K, Tojjar D, Yamada S, Toda K, Patel CJ, Butte AJ. Ethnic Differences in the Relationship Between Insulin Sensitivity and Insulin Response: A Systematic Review and Meta-Analysis. Diabetes Care (2013) 36(6):1789-96. doi: $10.2337 / \mathrm{dc} 12-1235$
34. Yabe D, Seino Y, Fukushima M, Seino S. Beta Cell Dysfunction Versus Insulin Resistance in the Pathogenesis of Type 2 Diabetes in East Asians. Curr Diabetes Rep (2015) 15(6):602. doi: 10.1007/s11892-015-0602-9

35. Beckman JA, Paneni F, Cosentino F, Creager MA. Diabetes and Vascular Disease: Pathophysiology, Clinical Consequences, and Medical Therapy: Part II. Eur Heart J (2013) 34(31):2444-52. doi: 10.1093/eurheartj/eht142

36. Cai X, Zhang Y, Li M, Wu JH, Mai L, Li J, et al. Association Between Prediabetes and Risk of All Cause Mortality and Cardiovascular Disease: Updated Meta-Analysis. BMJ (2020) 370:m2297. doi: 10.1136/bmj.m2297

37. Zheng H, Zhu H, Liu X, Huang X, Huang A, Huang Y. Mitophagy in Diabetic Cardiomyopathy: Roles and Mechanisms. Front Cell Dev Biol (2021) 9:750382. doi: 10.3389/fcell.2021.750382

38. Ma T, Huang X, Zheng H, Huang G, Li W, Liu X, et al. SFRP2 Improves Mitochondrial Dynamics and Mitochondrial Biogenesis, Oxidative Stress, and Apoptosis in Diabetic Cardiomyopathy. Oxid Med Cell Longev (2021) 2021:9265016. doi: 10.1155/2021/9265016

39. Wu J, Zheng H, Liu X, Chen P, Zhang Y, Luo J, et al. Prognostic Value of Secreted Frizzled-Related Protein 5 in Heart Failure Patients With and Without Type 2 Diabetes Mellitus. Circ Heart Fail (2020) 13(9):e007054. doi: 10.1161/CIRCHEARTFAILURE.120.007054

40. Ohkuma T, Ninomiya T, Tomiyama H, Kario K, Hoshide S, Kita Y, et al. BrachialAnkle Pulse Wave Velocity and the Risk Prediction of Cardiovascular Disease: An Individual Participant Data Meta-Analysis. Hypertension (2017) 69(6):1045-52. doi: 10.1161/HYPERTENSIONAHA.117.09097

41. Bressler P, Bailey SR, Matsuda M, DeFronzo RA. Insulin Resistance and Coronary Artery Disease. Diabetologia (1996) 39(11):1345-50. doi: 10.1007/ s001250050581

42. Cai X, Sun L, Liu X, Zhu H, Zhang Y, Zheng S, et al. Non-Alcoholic Fatty Liver Disease is Associated With Increased Risk of Chronic Kidney Disease. Ther Adv Chronic Dis (2021) 12:20406223211024361. doi: 10.1177/20406223211024361

43. Zhang X, Shu XO, Gao YT, Yang G, Li H, Zheng W. General and Abdominal Adiposity and Risk of Stroke in Chinese Women. Stroke (2009) 40(4):1098104. doi: 10.1161/STROKEAHA.108.539692

Conflict of Interest: The authors declare that the research was conducted in the absence of any commercial or financial relationships that could be construed as a potential conflict of interest.

Publisher's Note: All claims expressed in this article are solely those of the authors and do not necessarily represent those of their affiliated organizations, or those of the publisher, the editors and the reviewers. Any product that may be evaluated in this article, or claim that may be made by its manufacturer, is not guaranteed or endorsed by the publisher.

Copyright (c) 2022 Wang, Zheng, Li, Xu, Lu, Zhao, Li, Wang, Wang, Bi, Xu, Ning and Cai. This is an open-access article distributed under the terms of the Creative Commons Attribution License (CC BY). The use, distribution or reproduction in other forums is permitted, provided the original author(s) and the copyright owner(s) are credited and that the original publication in this journal is cited, in accordance with accepted academic practice. No use, distribution or reproduction is permitted which does not comply with these terms. 